\title{
Gestational Diabetes Mellitus (GDM) Related Common Risk Factors and Recurrence among Pregnant Patients: A Multifactorial Cross-Sectional Study
}

\begin{abstract}
Objective: To determine the frequency of newly diagnosed gestational diabetes mellitus (GDM) and common associated factors in recurrent GDM.

Study Design: Cross sectional.

Place and Duration of Study: The study was conducted in the department of Obstetrics and Gynecology unit A of Lady Reading Hospital, Peshawar from $27^{\text {th }}$ July 2016 to $27^{\text {th }}$ Jan 2017.

Materials and Methods: 336 women meeting the eligibility criteria (18-45 years, of any gestational age with gestational diabetes mellitus history and diagnosed as recurrent gestational diabetes) were included in the study. All patients who were labeled as a case of recurrent gestational diabetes was further investigated for any risk factor associated with its recurrence.

Results: The recurrence of Gestational Diabetes was observed in 99 (29.46\%) patients, in which $77 \%$ of patients had family history positive.

Conclusion: Keeping in view the recurrence of gestational diabetes proper screening and medical care for GDM in women during the childbearing years is highly recommended.
\end{abstract}

Key Words: Diabetes Mellitus (DM), Family History of GDM, Gestational Diabetes Mellitus (GDM), Pregnancy, Recurrence of GDM.

How to cite this: Tasneem S, Kashif S. Gestational Diabetes Mellitus (GDM) Related Common Risk Factors and Recurrence among Pregnant Patients: A Multifactorial Cross-Sectional Study. Life and Science. 2021; 2(2): 49-53. doi: http://doi.org/10.37185/LnS.1.1.131

This is an Open Access article distributed under the terms of the Creative Commons Attribution License (http://creativecommons.org/licenses/by/4.0), which permits unrestricted use, distribution, and reproduction in any medium, provided the original work is properly cited.

\section{Introduction}

Gestational diabetes mellitus (GDM)is classically been defined as any glucose intolerance identified during pregnancy. ${ }^{1}$ However, it has long been recognized that the diagnosis includes both categories i.e women who have diabetes before pregnancy but was not detected due to the lack of screening, as well as women with normal glucose levels before pregnancy, but got deteriorated during pregnancy. ${ }^{2}$ This definition includes women whose glucose intolerance will return to normal after pregnancy and those who will persist with glucose intolerance and type 2 diabetes. The latter group

${ }^{1}$ Department of Gynae and Obse/Anesthesia ${ }^{2}$

Frontier Crops Hospital, Quetta

Correspondence:

Dr. Saira Tasneem

Department of Gynae and Obse

Frontier Crops Hospital, Quetta

E-mail: drsairaakif13@gmail.com

Funding Source: NIL; Conflict of Interest: NIL

Received: Jul 17, 2019; Revised: Dec 27, 2020

Accepted: Mar 29, 2021 contains individuals who had unrecognized type 2 diabetes prior to pregnancy. ${ }^{3}$

Globally, Gestational Diabetes prevalence ranges between 1 and $14 \%$. $^{2}$ Women presented with diabetes during pregnancy, approximately $87.5 \%$ have gestational diabetes (which may or may not resolve after pregnancy), 7.5\% have type I diabetes and $5 \%$ have type II diabetes. Prevalence of diabetes mellitus type I and especially type II, has been increased in recent years. ${ }^{4}$ Incidence of gestational diabetes's has also been increased, due to increase obesity rate in population and advanced maternal age. The reported frequency of recurrent GDM varies widely, from 30 to $84 \%$, depending on the ethnicity of the subjects and diagnostic criteria. ${ }^{5}$

One of the major risk factors for developing GDM is previous history of pregnancy complicated by the disease. ${ }^{6}$ Other factors that have been identified as predictive of recurrent GDM include race/ethnicity, glucose tolerance test levels, early diagnosis of GDM during the initial pregnancy, need for insulin during the initial pregnancy, macrosomia during the initial 
pregnancy, advanced maternal age, maternal prepregnancy weight during the initial pregnancy, and an increase in pre-pregnancy weight between the initial and subsequent pregnancies, degree of hyperglycemia in index pregnancy, short interval between pregnancies, impaired fasting glucose and GTT (glucose tolerance test), 2 months postpartum. A study showed that in Korean women recurrence of GDM occur in nearly half of subsequent pregnancies and valuable clinical predictor of the risk of GDM is 2 months post-partum fasting blood glucose. ${ }^{7}$

A population based study, using hospital discharges, linked longitudinally and birth records (2001-2009) in New South Wales, Australia was carried out in 2015, concluded that incidence of gestational diabetes in the first pregnancy, was 3.7\% and in second pregnancy was $2.7 \%$. The rate of recurrence of gestational diabetes in a consecutive second pregnancy was $41.2 \%{ }^{8}$

A study published in Acta Obstetricia et Gynecologica Scandinavica December 2015, concluded that recurrence of diet treated GDM was $47.2 \%$ in primiparous women with previous history of GDM and the recurrence was associated with weight gain between pregnancies. ${ }^{9}$

A systematic review and meta-analysis, carried out on observational studies from 1973 to 2014, published in American Journal of Obstetrics and Gynaecology in September 2015, concluded that there is a significant association between ethnicity and gestational diabetes recurrence. According to the study, primiparous women and -non-Hispanic whites had a low recurrence rate as compare to other ethnicities and multiparous women. The overall gestational diabetes rate of recurrence is high $48 \%{ }^{10}$

There is no local data available regarding frequency of gestational diabetes among women with previous GDM history. The main purpose of the current study was to examine the rate of recurrence of gestational diabetes in a large group of women who had a history of GDM in the first pregnancy and to assess factors, associated with recurrence in a tertiary care hospital.

\section{Materials and Methods}

After approval from ethical review committee and informed patient's consent, study was carried out on a sample size of 336 patients (based on $30 \%$ prevalence, $95 \%$ confidence interval and $5 \%$ margin of error) ${ }^{5}$, in six months duration from $27^{\text {th }}$ July 2016 to $27^{\text {th }}$ Jan 2017, in Obstetrics and Gynaecology unit A, Lady Reading Hospital, Peshawar. Pregnant women, 18-45 years, of any gestational age with gestational diabetes mellitus history, were included by non-probability consecutive sampling. Patients with type I or type II Diabetes mellitus, polycystic ovarian syndrome and GDM diagnosed for $1^{\text {st }}$ time in this pregnancy, were excluded from the study. All women meeting the inclusion criteria were given a $75 \mathrm{~g}$ 2-hour OGTT, either in the first or second trimester after booking, and a further $75 \mathrm{~g}$ OGTT done at 24-28 weeks if the results of the first OGTT obtained, was found normal.

All patients who were labelled as a case of recurrent gestational diabetes were further investigated for any risk factor associated with its recurrence.

The statistical software for data analysis was SPSS version 16.0. Frequencies and percentages were calculated for qualitative variables like family history of diabetes, history of macrosomic baby, fasting plasma glucose level equal or more than $5.6 \mathrm{mmol} / \mathrm{L}$, 2 hours random blood glucose level equal or more than $7.8 \mathrm{mmol} / \mathrm{L}$ and BMI more than $30 \mathrm{~kg} / \mathrm{m} 2$. Mean +_S.D. were calculated for quantitative variables like age and period of gestation. Gestational diabetes was stratified among age groups, period of gestation, family history of diabetes, history of the macrosomic baby in a previous pregnancy and BMI values. Post-stratification chi-square test was applied keeping $p$-value $<0.05$ as significant. The results were presented as table and charts /graphs.

\section{Results}

There were, $n=155$ (46.13\%) patients in 18-30 years category and $n=181$ (53.86\%) patients in 31-45 category. Family history of diabetes $n=261$ (77.32\%) was the main risk factor in recurrent GDM. There were $n=99$ (29.46\%) patients with history of macrosomic babies. Patients with fasting plasma glucose $>5.6 \mathrm{mmol} / \mathrm{L}$ were $\mathrm{n}=75(22.32 \%)$. patients with random glucose level $>7.8 \mathrm{mmol} / \mathrm{L}$ were $\mathrm{n}=24$ (7.14\%) and patients with $\mathrm{BMI}>30 \mathrm{~kg} / \mathrm{m} 2$ were $\mathrm{n}=149$ (44.34\%) (Table 1). Mean and SD for age was 30 Years \pm 4.85 . Mean and SD for gestational age was 26 Weeks \pm 3.26 (Table 2). Prevalence of recurrent gestational diabetes was observed in $n=99(29.46 \%)$.

\section{Discussion}


Poorly controlled gestational diabetes is associated

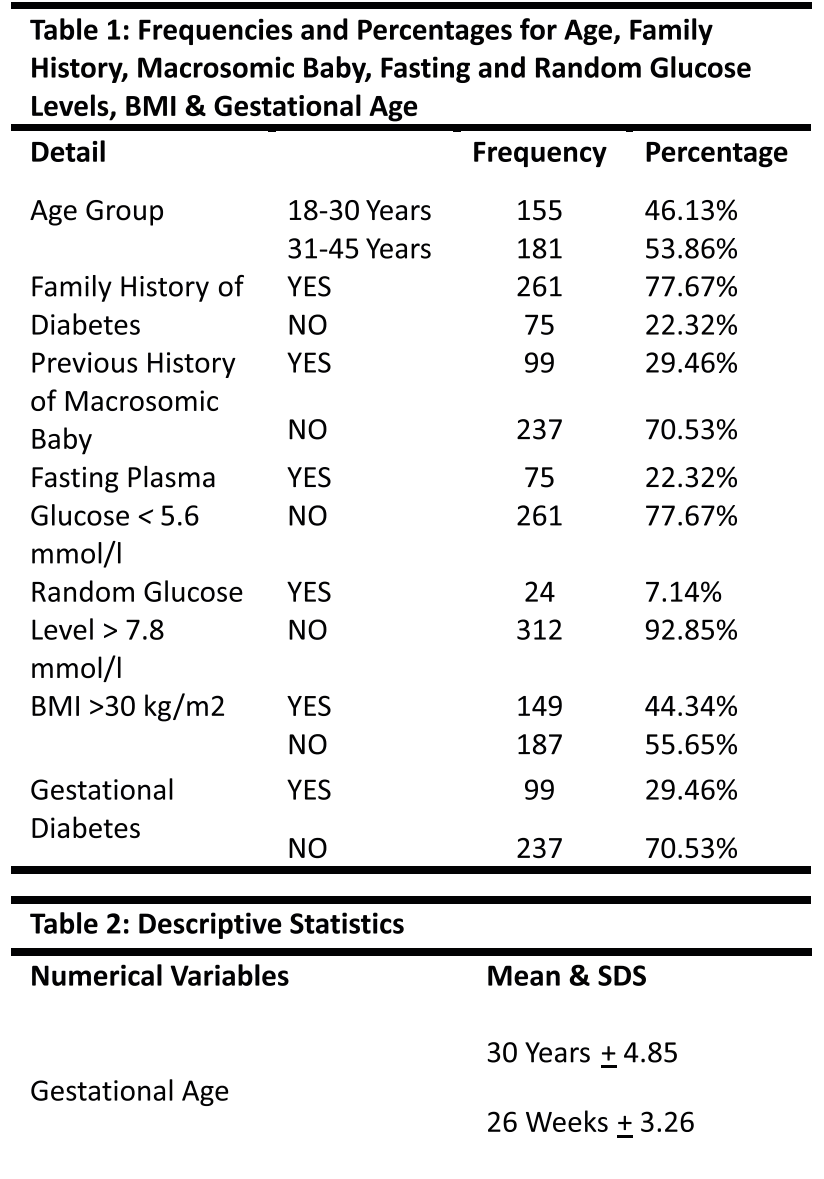

with adverse maternal, fetal and neonatal outcomes, including increased risk of pregnancy induced hypertension, pre-eclampsia and long-term risk of developing type 2 diabetes ( $50 \%$ risk in 5 to 10 years). Fetal and neonatal risks include risk of miscarriages, congenital structural anomalies, polyhydramnios, macrosomia, preterm labour and delivery, sudden intrauterine fetal deaths, birth trauma, shoulder dystocia, neonatal hypoglycemia, hypothermia, polycythaemia and hyperbilirubinemia. ${ }^{11}$

We studied the frequency of recurrent gestational diabetes and compared common risk factors associated with recurrence. Gestational diabetes prevalence is increasing at an alarming level in Pakistan. A progressive increase in the prevalence of GDM from the year 2005 till the year 2018 was observed in a tertiary care hospital of Karachi from $6.3 \%$ in 2005 to $19 \%$ in $2018 .{ }^{12}$ This increase in prevalence is partly due to performing universal GDM screening and adopting the International Association of Diabetes in Pregnancy Study Group
(IADPSG) criteria. However this increase in prevalence is a reflection of increasing back ground prevalence of Type 2 Diabetes Mellitus and obesity as well. Two recent surveys have shown the prevalence of Type 2 Diabetes in Pakistan at $26 \%$ and $17.8 \%$. The adverse maternal, fetal and neonatal outcomes can be prevented by improving screening and management strategies of GDM atearly pregnancy.

The frequency of recurrence of gestational diabetes is $26.46 \%$ in this study in contrast to $55 \%$ recurrence in a case control study conducted among primiparous women in China. ${ }^{13}$

Another study assessing the frequency of gestational diabetes showed 18.8 \% females with GDM have family history of diabetes which is comparable to our study results. However, previous history of GDM was present in only $6.8 \%$ of participants. ${ }^{14}$

Previous non-diabetic pregnancy with a macrosomic infant was found to be an independent risk factor for subsequent gestational diabetes mellitus that is in consistent to our study results. ${ }^{15}$

It is expected that the WHO 2013 diagnostic criteria may standardize GDM diagnosis worldwide, but to date, the implementation of these new criteria has been poor. There is limited data reflecting on the impact of the new diagnostic criteria on the gestational diabetes prevalence, amongst different ethnic groups. ${ }^{16} \mathrm{~A}$ Singaporean study showed that in the Asian population, the proportion of women diagnosed with GDM using WHO 2013 Criteria would be less however our study showed that gestational diabetes prevalence was observed in $n=99$ (29.46\%) patients which is quite high, mainly due to family history of GDM $n=261$ (77.67\%). ${ }^{17}$

The prevalence of GDM drops from $30.9 \%$ to $18.9 \%$ in Chinese and from $33.5 \%$ to $28.1 \%$ in South Asian women. ${ }^{18}$ while increase from $9.6 \%$ to $13.0 \%$. in Anglo-European population in Australia, The reason is differences in glycemic profiles on the OGTT from which GDM is diagnosed. ${ }^{19}$ In a group of over 850 GDM diagnosed women, from a multi-cultural community, in, Australia, the lowest fasting glucose levels ( $4.95 \pm 0.65 \mathrm{mmol} / \mathrm{L})$ was found in South-East Asia, with the highest 2 -h glucose level $(8.75 \pm 1.17$ $\mathrm{mmol} / \mathrm{L})^{20}$ which is comparable to our study. In a retrospective study on 24,325 patients, presenting at the University of San Francisco, using a BMI of $\geq 25$ as 
a screening tool, $76.8 \%$ of African-Americans and $24.9 \%$ of Asian women were diagnosed with GDM. Likewise using a BMI cut-off of $>21.0,91.5 \%$ of African American women, $90.1 \%$ of Hispanic, and $79.8 \%$ of United States Caucasian, but only $68.4 \%$ of Asian women were having GDM. African Americans were found to have the highest increased risk (OR: 5.1) of GDM when screening tool is BMI > 25.0, compared with Hispanics (OR: 2.7), Asians (OR: 2.3) and US Caucasians (OR: 3.6). ${ }^{21-24}$ Asian women were found to have GDM during pregnancy irrespective of having a BMI that is within or below normal range. Hunsberger et alfound that regardless of BMI greater or lesser than $26 \mathrm{~kg} / \mathrm{m}^{2}$.

Asian women had the greatest risk of having GDM as compare to other ethnic groups. ${ }^{25}$ This population tend to have more visceral or central fat, which is a known risk factor for insulin resistance and cardiovascular disease. Therefore, we would recommend screening of pregnant women for GDM irrespective of their BMI. A recent study on the interaction between $\mathrm{BMI}$ and maternal age, showed that odds ratios for GDM development were significantly higher in Caucasian women of more than 30 years, African of more than 25 years but older than 20 years if they were South-Asians. ${ }^{26}$ Another study found that Africans and South-Indians were at higher risk of having GDM irrespective of BMI. $^{27}$

The results of this study can guide us to develop strategies for management of these patients.

\section{Conclusion}

The results of the study warrants proper screening and medical care for GDM in women during the childbearing years.

\section{REFERENCE}

1. Kampmann U, Madsen LR, Skajaa GO, Iversen DS, Moeller N, Ovesen P. Gestational diabetes: a clinical update. World journal of diabetes. 2015; 6: 1065.

2. Kim C. Maternal outcomes and follow-up after gestational diabetes mellitus. Diabet Med. 2014; 31:292-01.

3. Rayanagoudar G, Hashi AA, Zamora J, Khan KS, Hitman GA, Thangaratinam S. Quantification of the type 2 diabetes risk in women with gestational diabetes: a systematic review and meta-analysis of 95,750 women. 2016; 59:1403-11.

4. Zhu Y, Zhang $C$. Prevalence of gestational diabetes and risk of progression to type 2 diabetes: a global perspective. Curr Diab Rep. 2016; 16: 7.

5. Kruse AR, Darling MS, Hansen MKL, Markman MJ,
Lauszus FF, Wieland HB. Recurrence of gestational diabetes in primiparous women. acta obstet gynecol scand. 2015; 94; 1367-72.

6. Duman NB.Frequency of gestational diabetes mellitus and the associated risk factors. Pak JMed Sci. 2015; 31: 194-7.

7. Schwartz N, Green MS, Yefet E, Nachum Z. Modifiable risk factors for gestational diabetes recurrence. Endocrine. 2016; 54: 714-22.

8. Schoenaker DA, Soedamah-Muthu SS, Callaway LK, Mishra GD. Pre-pregnancy dietary patterns and risk of gestational diabetes mellitus: results from an Australian populationbased prospective cohort study. Diabetologia. 2015; 58: 2726-35.

9. Kruse AR, Darling MS, Hansen MK, Markman MJ, Lauszus FF, Wielandt HB. Recurrence of gestational diabetes in primiparous women. Acta obstetricia et gynecologica Scandinavica. 2015; 94: 1367-72.

10. Schwartz N, Nachum Z, Green MS. The prevalence of gestational diabetes mellitus recurrence-effect of ethnicity and parity: a metaanalysis. American journal of obstetrics and gynecology. 2015; 213:310-7.

11. Basit A, Fawwad A, Qureshi H, Shera AS \& NDSP Members. Prevalence of diabetes, pre-diabetes and associated risk factors: second National Diabetes Survey of Pakistan (NDSP), 2016-2017. BMJ. 2018; 8: e020961.

12. Aamir AH, UI-Haq Z, Mahar SA, Qureshi FM, Ahmad I, Jawa $A$, et al. Diabetes Prevalence Survey of Pakistan (DPS-PAK): prevalence of type 2 diabetes mellitus and prediabetes using HbA1c: a population-based survey from Pakistan. BMJ Open. 2019; 9: e025300.

13. Wang YY, Liu Y, Li C, Lin J, Liu XM, Sheng JZ, et al. Frequency and risk factors for recurrent gestational diabetes mellitus in primiparous women: a case control study. BMC endocrine disorders. 2019; 19: 1-7.

14. Riaz M, Nawaz A, Masood SN, Fawwad A, Basit A, Shera AS. Frequency of gestational diabetes mellitus using DIPSI criteria, a study from Pakistan. Clinical Epidemiology and Global Health. 2019; 7: 218-21.

15. Rottenstreich $M$, Rotem $R$, Reichman $O$, Farkash $R$, Rottenstreich A, Samueloff A, et al. Previous non-diabetic pregnancy with a macrosomic infant - Is it a risk factor for subsequent gestational diabetes mellitus?. Diabetes Research Clinical Practice. 2020; 168: 108364.

16. Pu J, Zhao B, Wang EJ, Nimbal V, Osmundson S, Kunz L, et al. Racial/ethnic differences in gestational diabetes prevalence and contribution of common risk factors. Paediatric and perinatal epidemiology. 2015; 29: 436-43.

17. Chong YS, Cai S, Lin H, Soh SE, Lee YS, Leow MK, et al. Ethnic differences translate to inadequacy of high-risk screening for gestational diabetes mellitus in an Asian population: a cohort study. BMC pregnancy and childbirth. 2014; 14: 345.

18. Wang LF, Wang HJ, Ao D, Liu Z, Wang Y, Yang HX. Influence of pre-pregnancy obesity on the development of macrosomia and large for gestational age in women with or without gestational diabetes mellitus in Chinese population. Journal of Perinatology. $2015 ; 35$ : 985-90.

19. Wong VW, Lin A, Russell H. Adopting the new World Health Organization diagnostic criteria for gestational diabetes: How the prevalence changes in a high-risk region in 
Australia. Diabetes research and clinical practice. 2017; 129: 148-53.

20. Mukerji G, Kainth S, Pendrith C, Lowe J, Feig DS, Banerjee AT, et al. Predictors of low diabetes risk perception in a multi-ethnic cohort of women with gestational diabetes mellitus. Diabetic Medicine. 2016; 33: 1437-44.

21. Chang AL, Hurwitz E, Miyamura J, Kaneshiro B, Sentell T. Maternal risk factors and perinatal outcomes among pacific islander groups in Hawaii: a retrospective cohort study using statewide hospital data. BMC pregnancy and childbirth. 2015; 15: 239.

22. McDonald R, Karahalios A, Le T, Said J. A retrospective analysis of the relationship between ethnicity, body mass index, and the diagnosis of gestational diabetes in women attending an Australian antenatal clinic. International journal of endocrinology. 2015; 2015.

23. Nanditha A, Ma RC, Ramachandran A, Snehalatha C, Chan
JC, Chia KS, et al. Diabetes in Asia and the Pacific: implications for the global epidemic. Diabetes care. 2016; 39: 472-85.

24. Yuen L, Wong VW. Gestational diabetes mellitus: challenges for different ethnic groups. World journal of diabetes. 2015; 6: 1024.

25. Lin PC, Hung $\mathrm{CH}$, Chan TF, Lin KC, Hsu YY, Tzeng YL. The risk factors for gestational diabetes mellitus: a retrospective study. Midwifery. 2016; 42: 16-20.

26. Syngelaki A, Pastides A, Kotecha R, Wright A, Akolekar R, Nicolaides $\mathrm{KH}$. First-trimester screening for gestational diabetes mellitus based on maternal characteristics and history. Fetal diagnosis and therapy. 2015; 38: 14-21.

27. Silva-Zolezzi I, Samuel TM, Spieldenner J. Maternal nutrition: opportunities in the prevention of gestational diabetes. Nutrition reviews. 2017; 75: 32-50. 